\title{
Parallel EELS characterization of TiC commercial powder
}

\author{
A. Duarte-Moller, C. González-Valenzuela \\ Centro de Investigación en Materiales Avanzados, S. C. \\ Miguel de Cervantes 120, Complejo Industrial Chihuahua \\ Chihuahua, Ch. 31109, México
}

A commercial poiwder of $\mathrm{TiC}$ was characterized by EELS technique. EELS experiments were carried out in a CM 200 transmission electron microscope with a GATAN 766 PEELS attachment. A primary energy of $200 \mathrm{keV}$ and a spot size of $500 \mathrm{~nm}$ with an average acquisition time of 15 minutes were used. The atomic distribution was calculated performing the fast Fourier transform, FFT, on the core loss window, which is located at few eV beyond the core excitation Structural parameters were corrected with their respective experimental phase shifts and compared with those obtained by EXAFS, and the known crystallographic values. The extended fine structure energy range was taken at $300 \mathrm{eV}$ beyond the Ti L23 ionization edge which is located at an energy loss of $435 \mathrm{eV}$.

Figure 1 shows the energy loss spectrum in the low loss window for TiC system. As we can see, the most prominent plasmon loss peak characterizing TiC appears at an energy loss of $20.3 \mathrm{eV}$. This value is slightly different to that corresponding to metallic Ti. Close to bulk phsmon appears a small shoulder at an energy loss of $14.3 \mathrm{eV}$ which can be explained in terms of the surface effects. Las $\mathrm{t}$ supposition is possible thinking in that the thickness of the was of $450 \mathrm{~nm}$, corresponding at a mean free path of $0.85 \mathrm{~nm}$

The energy loss spectrum for the same material around the ionization Ti L23-edge and N K-edge corrected for background and multiple scattering process is shows in Figure 4. In this figure we can appreciate the finger print of the $\mathrm{TiC}$ structure. Also is noticeable the $\mathrm{C} \mathrm{K}$-edge which appears at an energy of $283.8 \mathrm{eV}$ which is shifted an approximated energy of $0.6 \mathrm{eV}$ respect to to the known value. Finally the $\mathrm{Ti}_{2} \mathrm{~L}_{23}$-edge appears at an energy loss of $464.3 \mathrm{eV}$, shifted an estimated energy of $8 \mathrm{eV}$ respect to the metallic Ti. Basically these differences can be explained in terms of the differences in their bond lengths

Figure 5 shows the radial distribution function obtained by applying the FFT (EXEELFS processing) to the $\mathrm{TiC}$ energy loss spectra in the window corresponding to the $\mathrm{TiL}_{23}$ fine structure. The structural parameter obtained have an estimated error less than $0.1 \%$, After to applying the experimental phase shift correction. This disagreement practically disappears when the experimental $\Delta R$ defined as $\Delta R=n \pi / 2 \Delta k$ was applied. Both results are in excellent agreement respect to the known crystallographic values. A comparison of the atomic distances for first and second nearest neighbors is showing in Table 1.

We conclude that the correction in RDF by phase shift and $\Delta R$ give an exact result in accordance with the respective known crystallographic values and in a very short time. These results establish the possibility to perform an excellent, reliable and precise way to obtain some physical properties of a wide number of materials. 


\section{References}

[1]. J. Pflüger, J. Fink, W. Weber and K. P. Bohnen, Phys. Rev. B. vol.30, No. 5 (1984) 1155

[2]. A. Duarte-Moller, L. Cota-Araiza, L. Morales de la Garza, G. A. Hirata, D. H. Galván, M. Avalos-Borja, Appl. Surf. Sci. 108 (1997) 59

[3]. Gilles Berhault, Apurva Mehta, Alexandru C. Pavel, Jianzhong Yang, Luis Rendón, Miguel José Yacamán, Leonel Cota Araiza, Alberto Duarte Moller and R. Chianelli, J. of Catalysis 198, (2001)9-19

Table 1. Numerical values for the atomic positions corresponding to the TiC structure.

\begin{tabular}{lcc}
\hline & $\begin{array}{c}\text { First nearest neighbors } \\
(\mathrm{nm})\end{array}$ & $\begin{array}{c}\text { Second nearest } \\
\text { neighbors } \\
(\mathrm{nm})\end{array}$ \\
\hline Without phase shift adjustment & 0.199 & 0.305 \\
With phase shift adjustment & 0.208 & 0.296 \\
Known crystallographic value: & 0.211 & 0.299 \\
\hline
\end{tabular}

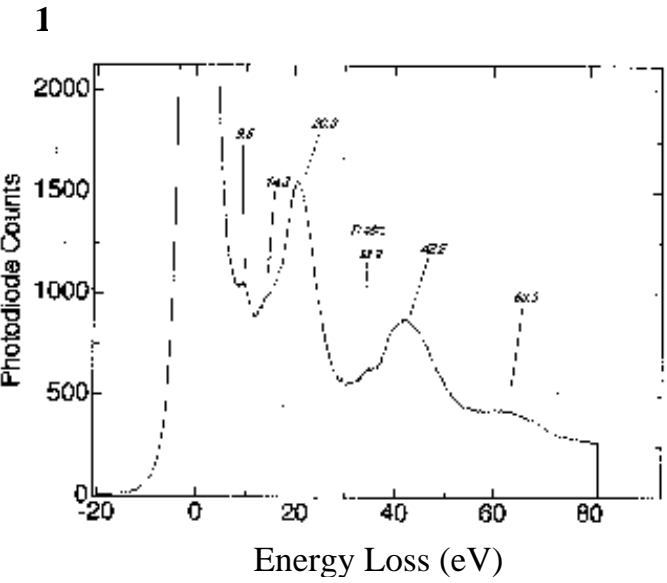

3

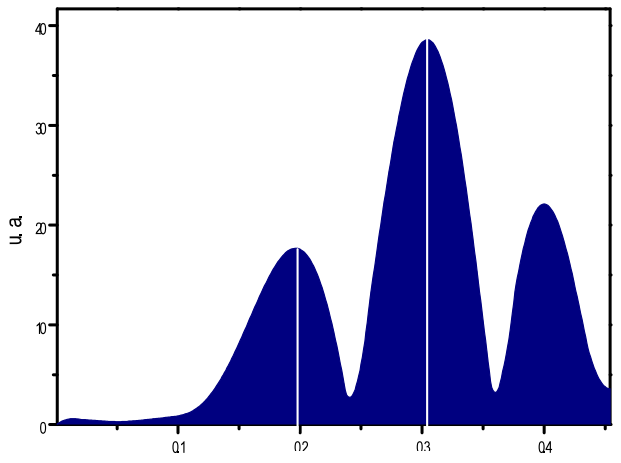

Radial distance
2

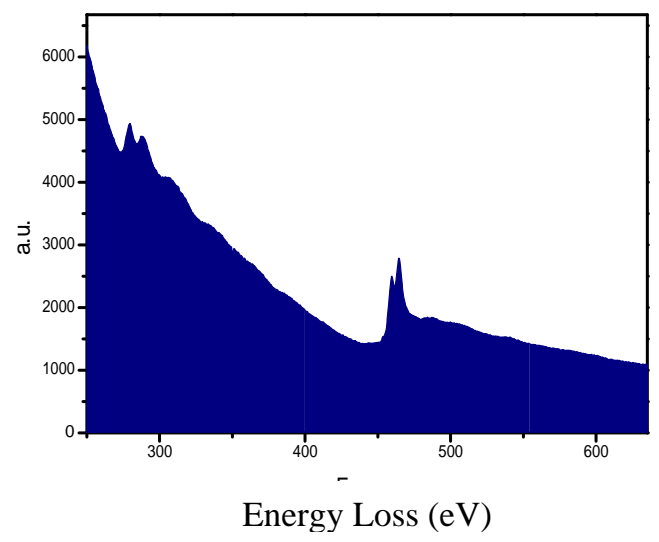

Fig1. Low loss window of TiC

Fig2. Core Loss window of TiC without background sustraction.

Fig 3. Radial distribution function obtained from Fig2. 